\title{
CompAr: \\ Ensuring Safe Around Advice Composition
}

\author{
Renaud Pawlak ${ }^{1}$, Laurence Duchien ${ }^{1}$, and Lionel Seinturier ${ }^{2}$ \\ 1 Université de Lille, INRIA Futurs-Jacquard \\ Bâtiment M3, Villeneuve d'Ascq, 59655, France \\ 2 Université de Paris 6, LIP6, INRIA Futurs-Jacquard \\ 4, place Jussieu, 75252 Paris, France
}

\begin{abstract}
Advanced techniques in separation of concerns such as Aspect-Oriented Programming, help to develop more maintainable and more efficient applications by providing means for modularizing crosscutting concerns. However, conflicts may appear when several concerns need to be composed for the same application, especially when dealing with around advice. We call this problem the Aspect Composition Issue (ACI). Based on our experience in programming aspects, this paper presents a language called CompAr, which allows the programmer to abstractly define an execution domain, the advice codes, and their execution constraints. The CompAr compiler then evaluates the definitions in order to check if the execution constraints are fulfilled. Using a concrete AOP case study, we show how to use the CompAr language in order to detect and avoid ACIs.
\end{abstract}

\section{Introduction}

When dealing with complex software, programmers and designers naturally try to apply the divide and conquer principle by splitting the application into small pieces, which are easier to understand than the whole system. This technique is referred to as Separation of Concerns (SoC) and has been originally described in $[5,13]$. The goal of SoC is to analyze one of the parts without having to take the other parts into account. However, in the difficult process of making the application parts independent, many issues can arise.

Within the last few years, Aspect-Oriented Programming (AOP) [9] has stressed the point that some concerns are significantly difficult to modularize. AOP identifies these concerns as crosscutting concerns, i.e. the implementation of these concerns spans over some modules of the other concerns. AOP and related approached propose some solutions to these issues which would pull out the crosscutting concerns from the application code, allowing for easier modularization.

Thanks to AOP, some techniques that are used in middleware and other fields have been highlighted and are becoming more popular. One of the most important and widely used techniques can be referred to as the around advising of the code; this is an important mechanism used to compose concerns together. It 
has been employed under several contexts and can be implemented by wrappers, filters, interceptors, proxies, around code injections, and so on. However, all these implementation techniques face the same issues when composing several concerns. We call this kind of issue the Aspect Composition Issue (ACI) [14]. Unfortunately, little support is provided to solve this problem and, most of the time, it has to be handled manually, without any tools or guidelines.

In this paper, we present a language called CompAr that is able to automatically detect conflicting around advice codes out of an abstract specification of the aspect-oriented program.

In section 2, we define the around advice and give some examples of its use. Then, in section 3, we present our case study, which deals with real-life aspects that we use for reasoning on specific ACIs. In section 4, we define CompAr, our language to specify composition-relevant information. We show how CompAr checks a specification to detect composition issues. Section 5 goes further in studying ACI by focussing on composing all the aspects of our case study by using CompAr. Finally, before concluding, we list some related works in section 6 .

\section{The Around Advice}

Breaking down software into independent modules, objects or components that can be designed or programmed separately, implies a composition phase. In this section, we focus on a useful composition mechanism called around advice; we introduce its mechanisms and common utilizations.

\subsection{Introducing Around Advice}

When composing several modules together, structural or behavioral composition mechanisms are needed; our focus is on the behavioral compositions. When composing behaviors, the behavior of the target module is modified by another behavior coming from source modules. In order to achieve this, around advice is a convenient mechanism; we describe the device here in an informal manner.

One can apply an around device code to a given target executable element such as a method, a constructor, or a field access; as a result, the target element will be modified transparently for the base program; the target element is said to be advised. Once this element is executed in the program, the flow of execution is the following:

1. the advice code is executed (and can access some contextual information from the base program),

2. when a special instruction called proceed is reached, the advice code executes the advised executable element (called this),

3. when the advised method ends, the rest of the advice code is executed and can finally return.

Note that an advice code has a before part (before the call to proceed) and an after part (after the call to proceed). It is not required to call proceed (it then completely replaces the implementation of the advised method). 


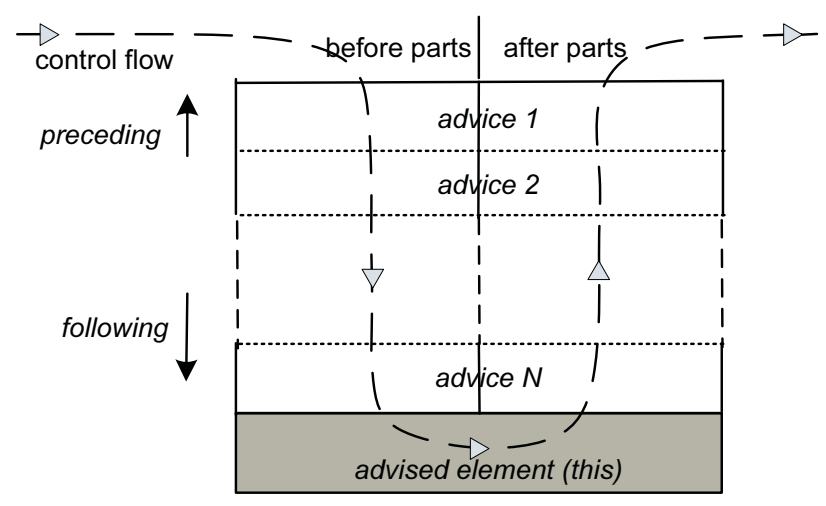

Fig. 1. An around advice chain.

As shown in figure 1, a method can be advised several times; this method then holds an advice chain. Each advice before part is executed in the order defined by the chain (the top advice code is executed first). When the execution of an advice code reaches proceed, the control is passed to the next advice of the chain.

The advised element is executed at the end of the chain and returns so that all the advice after parts are executed in the reverse order of the before parts execution order. An advice code can break the regular advice chain control flow by not calling proceed or by throwing an exception.

Note that an advice code is said to precede the advice codes that come after it in the chain and to follow the advice codes that come before it in the chain.

\subsection{Around Advice Utilizations}

Even though around advice-related techniques are used in many contexts and languages, the main utilization of around advice is in Aspect-Oriented Programming (AOP) [9]. AOP focuses on solving crosscutting of concerns when programming or designing complex applications. In short, AOP solves crosscutting and tangling code issues by allowing the programmer to define pointcuts. A pointcut is a set of points (joinpoints) in the base program that are affected by aspect-level advice, including around advice.

Aside from the popular AspectJ [8] language, numerous projects have included AOP features such as JAC [15], CFOM [2], CaesarJ [11], and PROSE [16]. Besides, several other approaches use techniques that can be closely related to around advising, for instance: Composition Filters [2] and Multi-Dimensional Separation of Concerns [12].

When a new language is not available, around advice is usually implemented by using Interception [10] [15], which is a very popular mechanism to implement separation of concerns in middleware environments. In these frameworks, interceptors are regular objects which may intercept the method invocations, 
the object constructions, and/or the field accesses. Chains of interceptors are functionally equivalent to around advice chains.

The following code ${ }^{1}$ shows the implementation of an interceptor using the AOP-Alliance [1] Java interfaces (the common Public Domain interfaces that have been defined and implemented by some of the the aforementioned projects).

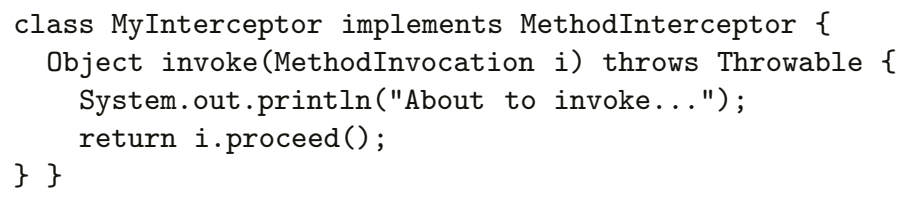

\section{An AOP Case Study for Composition}

This section presents a set of useful generic server-side aspects which are typically used in distributed middleware layers.

For the sake of this paper, we have focused solely on the around devices of the aspects, and have simplified them to keep only the relevant details. Note that we use the AOP Alliance API in order to remain as independent as possible from any specific language or commercial framework. The aspects depicted here are used to illustrate typical composition issues, and they will be formalized in the next sections.

\subsection{Logging Aspect}

The most well-known and straightforward application of AOP consists of seamlessly introducing logging when needed. By using around advice, the logging aspect can write into files what happens on a server; this can be useful for maintenance (security, performance, debugging). As shown below, the implementation of the logging aspect's around advice is quite simple.

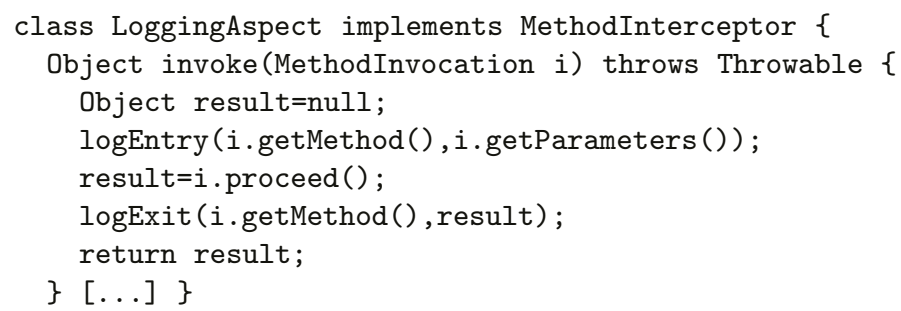

\subsection{Authentication Aspect}

Within a client/server interaction, a server-side authentication aspect checks that the user associated to the current session has the right to access the involved

\footnotetext{
${ }^{1}$ Note that the throws Throwable clause is used since proceed throws a Throwable and that the interceptors most of the time forward the exceptions rather than handling them.
} 
resources. If the current session has no associated user, the authentication aspect may ask the client to authenticate by, for example, asking for a login and a password. If the client does not have the right to perform the current action, the authentication aspect performs an alternative action, such as throwing an exception to notify the client that the rights were not granted.

The implementation of the authentication aspect's logic is mainly done within the following around advice:

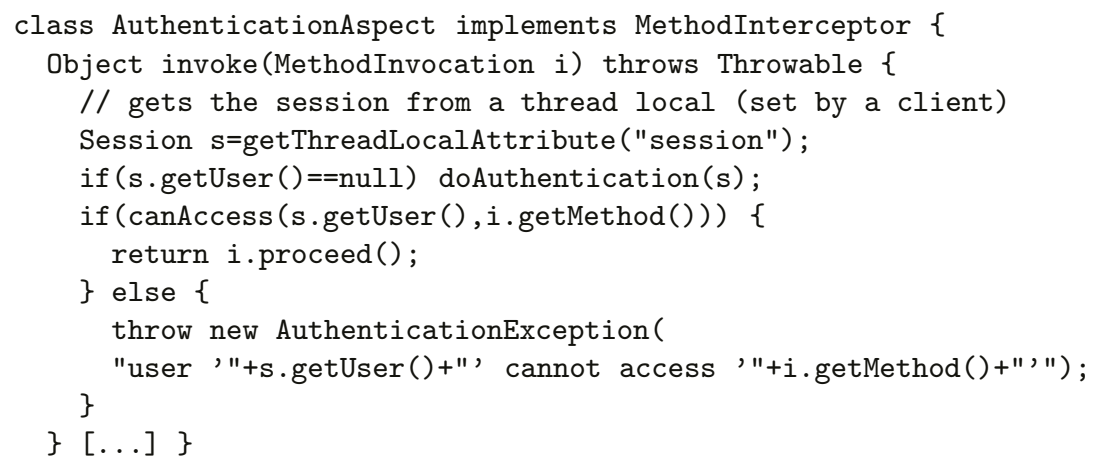

\subsection{Persistence Aspect}

On the server, objects can be persistent. Typically, this is achieved by advising all the setters and getters of the objects and by writing or reading the data in a storage (XML files, JDBC data source). In many systems, the object's fields may still be directly accessed on optimization purpose so that the object acts as a cache for the storage. Additionally, any transient object that is referenced by a persistent object (through a reference or a collection) should itself become persistent.

The implementation of the persistence aspect's logic is mainly done within the following around advice:

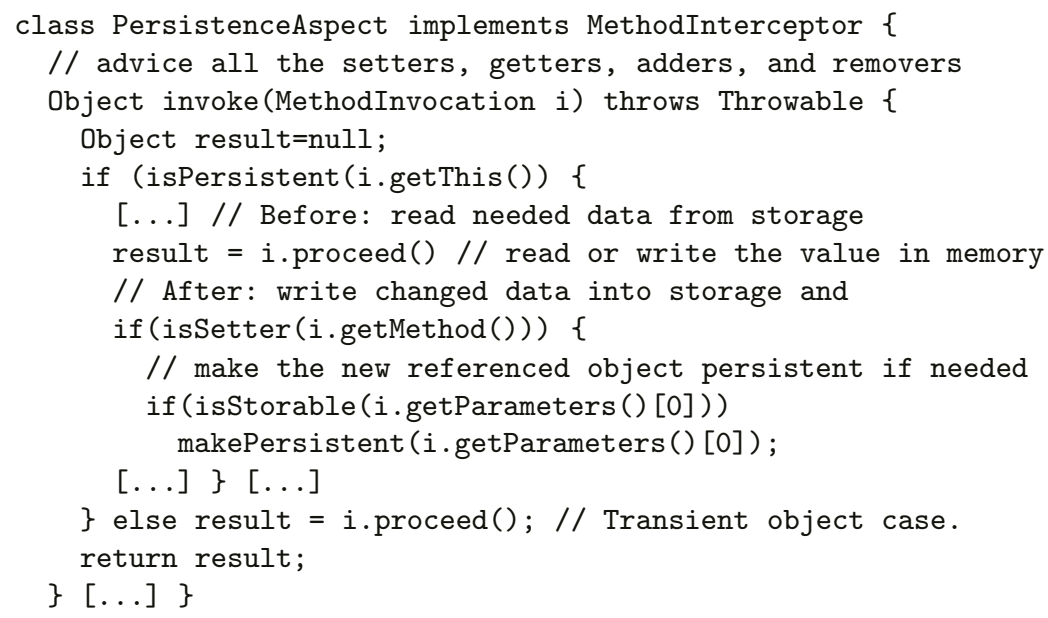




\subsection{Association Aspect}

In object or component models, entities may be related to each other through references or collections. At a higher level, these references or collections can be part of an association; they are then called roles.

For instance, an association exists between an employee and a company: an employee belongs to a company and a company employs several employees; each class (Employee and Company) defines a role field of this association. When a role that is part of an association is set, as through a role setter, it generally means that the other role should be updated in order to preserve the association integrity.

With AOP, it is possible to handle the association integrity concern within an aspect. This concern, which is usually a crosscutting one, can then be cleanly modularized and the maintenance of the application is more straightforward. The main logic of the association integrity concern is programmed in the following around advice:

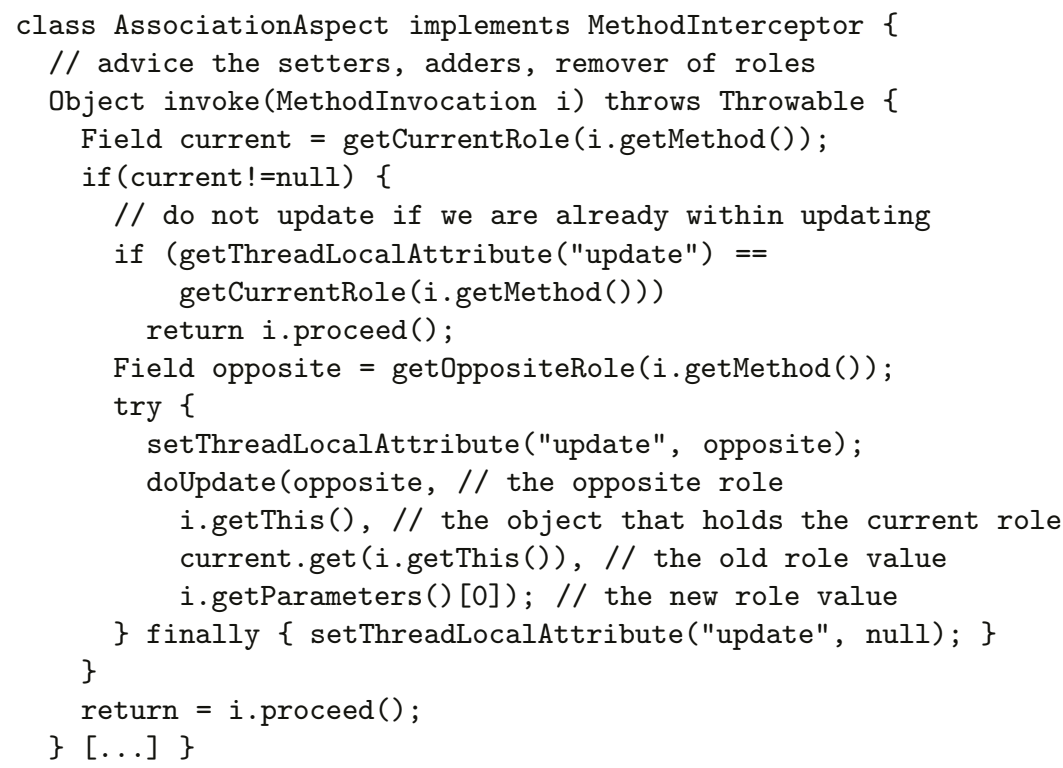

\subsection{Composing Logging, Authentication, and Persistence}

As a first introduction to ACIs (Aspect Composition Issues), this section informally shows how to compose the Logging, Authentication, and Persistence aspects. This simple composition problem illustrates the importance of correctly ordering the around advice.

When composing these three aspects, a simple reasoning can help the programmer to find the aspect interactions and thus find out how to solve them. Let us first look at the code of these aspects (see sections 3.1, 3.2, and 3.3). A quick glance shows that the only aspect that does not call proceed all the time 
is the authentication aspect. This property is important because when an aspect is programmed independently from any context, the programmer assumes that the invocation is actually proceeded to the advised element.

Therefore, if the logging around advice is placed after the authentication advice, then the logging will only be performed if the invocation is authenticated. However, it is not always the behavior that a programmer would expect for the system. Indeed, to detect attack attempts on the server, we may want to log all the requests, even if the associated action is not successfully executed. In contrast, a quick study of the persistence aspect shows that we want to apply the persistence only if the action is successfully executed.

Finally, we are in the presence of three significantly different kinds of around advice.

- The logging has an execution constraint which states that its before part must always be executed. By using first order action logic, this constraint can be expressed as: beforeLogging, where beforeLogging is true if the beforelogging part has been executed at the end of the advice chain execution. We refer to this kind of advice as obligatory advice.

- The persistence has an execution constraint which can be expressed as: [persistent]?this<=>writeStorage:true, to be read as "if our execution context is persistent (boolean), then the execution of this advised implies the execution of the writeStorage action and vice versa, else the execution constraint is always fulfilled (true)". Note that we refer to this kind of advice as exclusive advice.

- The authentication does not have an execution constraint, but it does not always call proceed. We refer to this kind of advice as conditional advice.

Taking into account all that has been said, in order to fulfill the obligatory and exclusive constraints, the best order for the chain should be: (Logging > Authentication > Persistence). Note that we have already informally presented this classification in [14]. This is, on the other hand, a very intuitive result that would need to be validated. Besides, when the number of aspects grows, it can become tedious to manually fulfill all the execution constraints. In the next section, we present CompAr, a language which helps the programmer to find and validate the right composition order in a rigorous way.

\section{Supporting Aspects Composition: The CompAr Language}

In the previous section, we presented a set of useful aspects for server-side middleware layers: Logging, Authentication, Persistence, and Association. Thanks to AOP and around advising, we have been able to separately define these different concerns so that the understanding of the sever-side system is easier. However, as seen in section 3.5 some ACIs are likely to appear when composing the aspects. This is due to the fact that each aspect is programmed independently, and holds some implicit constraints. 
In this paper, we go far beyond our first paper on the subject [14], which informally introduced the advice types and stated the ACI in a general way. Here, in order to deal with the ACIs, we have defined a language called CompAr (for Composing Around advice). CompAr allows the programmer to specify the advice codes and their implicit constraints. In addition, CompAr checks that a given composition order is valid for a set of execution contexts.

In the rest of this section, we present CompAr (4.1), we define its semantics (4.2), and we apply it to our composition example (4.3).

\subsection{The CompAr Language}

In order to introduce CompAr, we first show how to specify the composition problem which was informally presented in 3.5. In order to do this, we write the following abstract program:

choices: persistent, authenticated;

advice logging:loggingEnter \{loggingEnter+loggingExit\}

advice authentication \{ [authenticated]?-+-:throw NotAuthenticated \}

advice persistenceSetter:[persistent]?this<=>writeStorage:true \{

[persistent] ?-+(caller (persistent=true), writeStorage):-+-\}

advised a \{logging, authentication, persistenceSetter; \}

Where the choices command defines the different boolean variables of the execution domain, advice defines a new abstract advice code, and advised defines a composition order to be tested by the compiler. When run, the compiler executes the defined advised in the domain (all the possible combinations of choices values) and checks that the advice definitions are valid. Note that choices can be initialized to true or false in order to restrain the execution domain, but they are usually left undefined, as in this case, to test all the possible executions.

An advice definition contains two parts: an optional post-execution constraint, defined after the advice name and separated from it by a colon; and a body, within curly brackets, which represents the abstract definition of the advice code.

As a result, with CompAr, the logging advice programmed in section 3.1 can be abstractly defined by:

advice logging:loggingEnter\{loggingEnter+loggingExit\}.

It means that the body is composed of a loggingEnter before-proceed action and a loggingExit after-proceed action. Besides, when the advice is included in an advised, the post-condition execution constraint ensures that the loggingEnter action has been executed.

The persistence advice is more complicated but follows the same principle:

advice persistenceSetter: [persistent]?this<=>writeStorage:true $\{$ [persistent] ?-+(caller (persistent=true), writeStorage):-+- $\}$

As an execution constraint, we recognize the constraint defined in section 3.5. The body must be understood as follows: "If the execution context is persistent we define a body that does nothing as a before part and that executes two actions as an after part: (1) it sets the calling context to persistent (as an effect, 
the newly referred object is made persistent), (2) it executes the writeStorage action. If the execution context is not persistent, we just proceed the execution."

Note that we use - to indicate that the advised body code performs some action that is not relevant for composition.

\subsection{The CompAr Semantics}

We now give a brief overview of the CompAr semantics by using a denotational semantics. CompAr can be split into two sub-languages: the body language and the constraint language.

To help the semantics understanding, note that the body language syntax is given by the B rule (terminals are lower-cased identifiers and symbols other than 'I', ';', and ': :='):

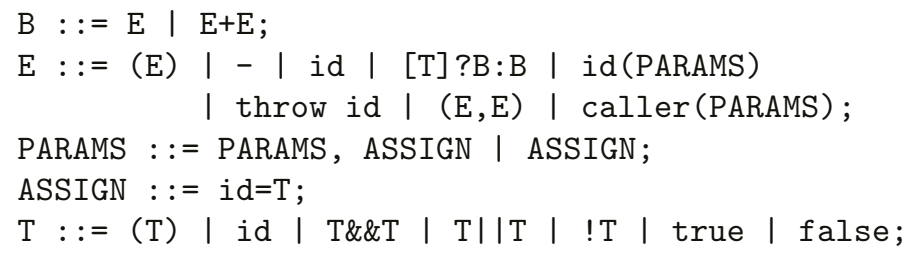

The constraint language syntax is defined by C:

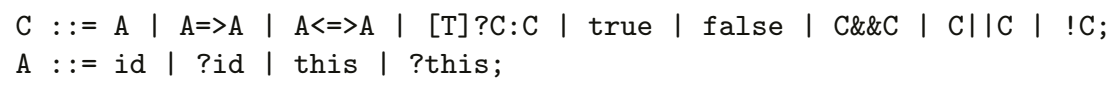

The Body Language Semantics: The body language is inductively defined

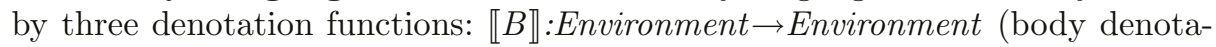

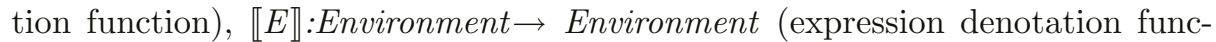
tion), and $\llbracket T \rrbracket:$ Environment $\rightarrow$ Boolean (boolean expression denotation function). For the sake of simplification, we use primitive functions that we informally describe.

The $\llbracket B \rrbracket$ denotation function is defined through the $\llbracket E \rrbracket$ denotation function:

1. $\llbracket E 1+E 2 \rrbracket(e)=\llbracket E 2 \rrbracket(\operatorname{proceed}(\llbracket E 1 \rrbracket(e)))$

2. $\llbracket B \rrbracket(e)=\llbracket E \rrbracket(e)$

where proceed:Environment $\rightarrow$ Environment is the function that corresponds to the denotation function of the next advice body in the chain. For a given advised execution, an environment contains a linked code list, which corresponds to the advice order that has been defined for the advised. Moreover, when the end of the chain is reached and the advised element is executed, a this action is set to 'executed' in the environment.

The $\llbracket E \rrbracket$ denotation function (for expressions) is defined as follows and using $\llbracket B \rrbracket$ and $\llbracket T \rrbracket$ : 
1. $\llbracket-\rrbracket(e)=e \quad / /$ skip function

2. $\llbracket(E) \rrbracket(e)=\llbracket E \rrbracket(e)$

3. $\llbracket(E 1, E 2) \rrbracket(e)=\llbracket E 2 \rrbracket(\llbracket E 1 \rrbracket(e))$

4. $\llbracket i \rrbracket(e)=e[$ executed $/ i]$

5. $\llbracket[T] ? B 1: B 2 \rrbracket(e)=\llbracket B 1 \rrbracket(e)$ if $\llbracket T \rrbracket(e)=$ true,$\llbracket B 2 \rrbracket(e)$ otherwise

6. $\llbracket i(i 1=T 1, \ldots, i n=T n) \rrbracket(e)$ $=\operatorname{advised}(i$, new $/ \llbracket T 1 \rrbracket(e) / i 1, \ldots, \llbracket T n \rrbracket(e) /$ in $], e[$ invoked $/ i \rrbracket)$

7. $\llbracket \operatorname{caller}(i 1=T 1, \ldots, i n=T n) \rrbracket(e)=\operatorname{parent}(e)[\llbracket T 1 \rrbracket(e) / i 1, \ldots, \llbracket T n \rrbracket(e) / i n \rrbracket$

where advised:Identifier $\times$ Environment $\times$ Environment $\rightarrow$ Environment is the function that initializes a new child environment (new: $\rightarrow$ Environment) with the linked code that corresponds to the advised identified by $i$. It then proceeds the first linked code of the chain in the new environment. Note that 'advised' sets the $i$ action state to 'invoked' in the parent environment. This $i$ action state will be set to 'executed' in the parent environment by the 'proceed' function whenever the advised element is executed in the child environment.

Note that two types of environment changes can be performed: an action state change, where an action can be set to 'executed' or 'invoked', and a choice boolean value assignment. This assignment can be done in a new context, during an 'advised' invocation, or in a calling context, during a caller instruction.

We do not formally define the throwing of exceptions since it is easy to understand intuitively. When a exception is thrown, the program terminates its execution and the environment is returned as is.

Finally, we do not define the denotation function $\llbracket T \rrbracket$ because it is a classical boolean expression denotation function.

The Constraint Language Semantics: When a CompAr program is executed, it is important to note that some choices may be left undefined. As a consequence, the compiler creates all the possible environments in order to cover the domain and check out all the possible executions. For instance, when an invocation towards an advised is done, a new set of environmental contexts is created and the invocation is performed for all these contexts. Hence, the compiler creates an execution tree rather than a simple execution.

When the execution tree is created, the compiler inspects all the final environmental contexts (one per tree node) and checks, for each one, that the advice post-execution constraints are fulfilled. For a given environment, we define the $\llbracket C \rrbracket$ denotation function, which is used for constraint verification.

1. $\llbracket[T] ? C 1: C 2 \rrbracket(e)=\llbracket C 1 \rrbracket(e)$ if $\llbracket T \rrbracket(e)=$ true, $\llbracket C 2 \rrbracket(e)$ otherwise

2. $\llbracket i \rrbracket(e)=$ true if $\operatorname{get}(i)(e)=$ executed, false otherwise

3. $\llbracket i \rrbracket(e)=$ true if $\operatorname{get}(i)(e)=$ invoked, false otherwise

4. $\llbracket C 1=>C 2 \rrbracket(e)=\llbracket C 1 \rrbracket(e) \Rightarrow \llbracket C 2 \rrbracket(e)$

5. $\llbracket C 1<=>2 \rrbracket(e)=(\llbracket C 1 \rrbracket(e) \Rightarrow \llbracket C 2 \rrbracket(e)) \wedge(\llbracket C 2 \rrbracket(e) \Rightarrow \llbracket C 1 \rrbracket(e))$

... the rest is regular boolean expressions 


\subsection{Testing Our Case Study with CompAr}

Note that the CompAr language and all the examples used in this paper are available for download at [4].

If we compile the program defined in section 4.1 , we can check that our informal reasoning led in section 3.5 is correct. The compiler then writes out:

[START] checking 'a' advised execution constraints...

[OK] [0] \{persistent=true, authenticated=true\}

[OK] [0] \{persistent=true, authenticated=false

[OK] [0] \{persistent=false, authenticated=true

[OK] [0] \{persistent $=f$ alse, authenticated $=f a l s e\}$

[END] no composition errors found while checking 'a'

As we can see, the compiler checked the post-execution constraints for the domain, which is formed out of the combination of the persistent and authenticated choices possible values. Here, since the values are undefined, there are four possible executions and they all fulfill the constraints for the advised: ordered as logging, authentication, persistenceSetter.

Next, if we try an invalid order such as persistenceSetter, authentication, logging, the compiler reports an error for each execution that does not fulfill all the constraints:

[START] checking 'a' advised execution constraints...

[OK] [0] \{persistent=true, authenticated=true\}

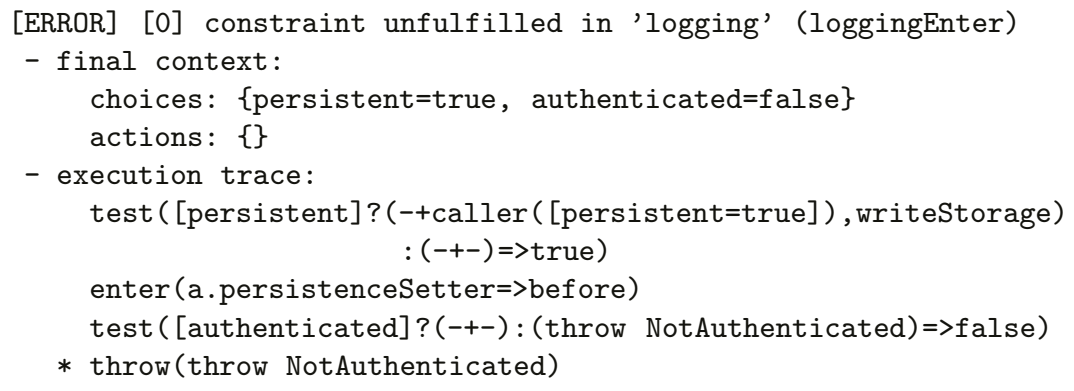

[OK] [0] \{persistent=false, authenticated=true

[ERROR] [0] constraint unfulfilled in 'logging' (loggingEnter) $[\ldots]$

[END] 2 composition error(s) found while checking 'a'

Here, we see that the loggingEnter constraint defined by the logging advice is not fulfilled when authenticated=true.

\section{Using CompAr to Solve Complex ACIs}

In this section, we finalize our case study which had started in sections 3.5 and 4.3 by adding the association aspect of section 3.4. As we will see next, adding 
this final aspect induces a difficult ACI that we manage to detect and solve with CompAr.

\subsection{Composing the Association}

For the association, the advice code, applied on a role setter, is active only if the advised method is a role (part of an association), and if we are not already in an update process. Further, in the case that the role\&\&! update condition is fulfilled, the association is exclusive - the opposite role of an association has to be updated only if the current role itself is updated. As a consequence, a possibly valid order for the roleSetter advice can be defined in the total advised: advised total \{ logging, authentication, persistenceSetter, roleSetter; \}.

More precisely, by using CompAr, the association around advice can be abstractly specified as:

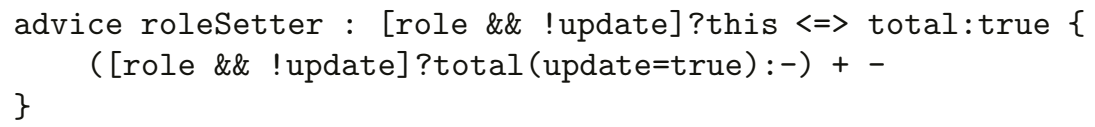

where total is the name of the advised that defines the full order of our four aspects. The association specification should be read as follows: "the before part does nothing except proceeding; the after part invokes recursively the advised total if we are in a role and not in an updating process (role \&\& !update condition)".

Note that this definition makes the total advised definition recursive. The infinite recursion is avoided by the update=true assignment which restricts the domain of the total invocation and prevents having to re-apply the roleSetter advice.

However, the total ordering leads to a conflict that we explain in the next section.

\subsection{The Persistence and Association Conflict}

Let us imagine that we want to apply our aspects to two objects o1 and o2, where 01 and $\circ 2$ can be linked through an association. This association has two roles $r 1$ and $r 2$. A method setR2 can be called on $\circ 1$ in order to set the association's role and a method setR1 can be called on 02 in order to set the association's opposite role. Let us also assume our initial conditions imply that $\circ 2$ is persistent, that $\circ 1$ is not persistent, and that $01 . r 2$ and $\circ 2 . r 1$ are null.

Figure 2 shows the execution flow when setR2(o2) is invoked on 01 and when the advice chain is the one suggested by the total advised of the previous section. Note that $\mathrm{x}$ refers to a memory variable, whereas $\underline{x}$ refers to the corresponding variable in the persistent storage.

As seen in the figure, the composition of the aspects as they are produces a side-effect that breaks the implicit persistence constraint; the final storage state 


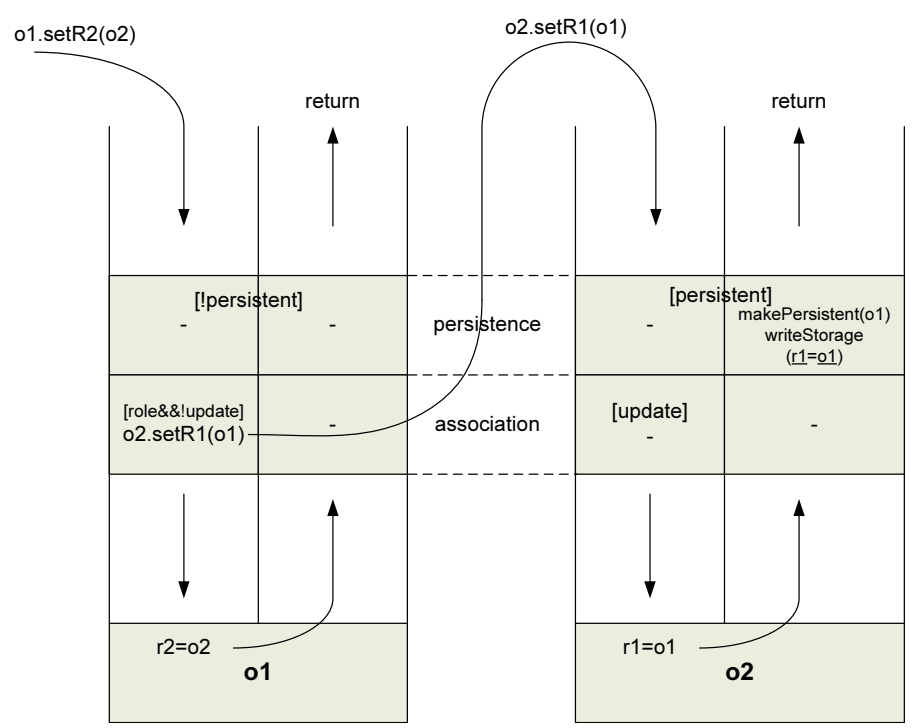

Fig. 2. Example of conflict between persistence and association.

$(\underline{\mathrm{o1}} \cdot \underline{\mathrm{r} 2}=\mathrm{null})$ differs from the final memory state $(\mathrm{o} 1 . \mathrm{r} 2=02)$. This composition error is mainly a result of the condition [persistent] being global to the before and after parts of the persistent advice code. As a consequence, the persistence after code, which is supposed to write the value of the $r 2$ role in the storage, is never executed.

\subsection{Solving the Conflict by Using CompAr}

In the previous section, we have seen that the persistence and the association conflict. Detecting this conflict requires a great deal of analysis and understanding from the aspect designer. However, by using CompAr, this conflict can be automatically detected. In fact, if we run CompAr on the total advised as defined in section 5.1, it gives the following output:

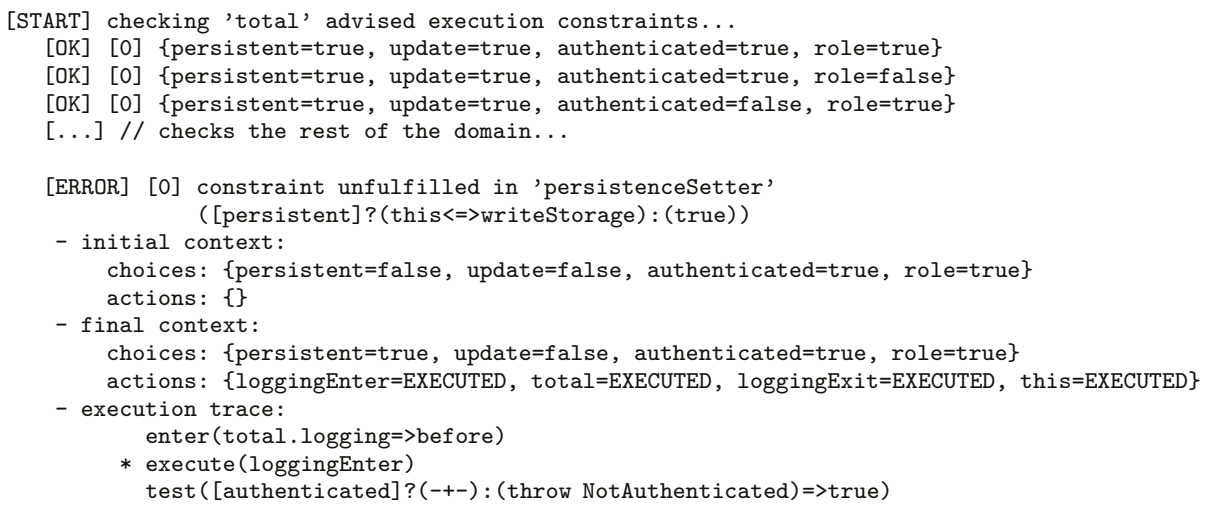




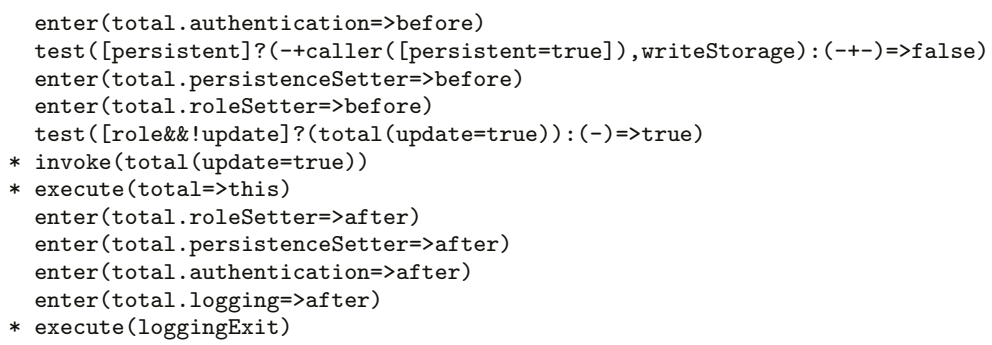

[OK] [0] \{persistent $=f$ alse, update $=f$ alse, authenticated $=$ true, role=false

[OK] [0] \{persistent $=f a l s e, ~ u p d a t e=f a l s e, ~ a u t h e n t i c a t e d=f a l s e, ~ r o l e=t r u e\}$

[OK] [0] \{persistent $=f a l s e, ~ u p d a t e=f a l s e, ~ a u t h e n t i c a t e d=f a l s e, r o l e=f a l s e\}$

[OK] [1] \{persistent=true, update=true, authenticated=true, role=true

[OK] [1] \{persistent=true, update=true, authenticated=true, role=false

[OK] [1] \{persistent=true, update=true, authenticated $=$ false, role=true

[...] // checks the rest of the domain for recursion level $1 \ldots$

[END] 1 composition error(s) found while checking 'total'

Therefore, by simply analyzing the compiler's output, the designer can deduce that the persistence execution constraint is not fulfilled because the action writeStorage is not executed at level 0 of the recursive evaluation (the final context contains a list of the executed actions). A simple solution to solve this conflict is to decouple the [persistent] condition. In fact, by specifying the persistence body as, ([persistent] ?-:-)+([persistent] ? (caller (persistent= true), writeStorage):-), the compiler does not report any more errors. It is then easy for the designer to report this design change in the persistence implementation of section 3.3.

\section{Related Works}

Some important studies on aspect interaction are conducted in [6]. This work, which is based on a more precise definition of the AOP's pointcuts semantics, allows their authors to automatically detect the points where a potential conflict may occur (the points where several advice codes are applied). However, it does not give solutions for ordering the advice codes. We believe that this work and our work are complementary.

As shown in [7], superimpositions define program modules that can augment distributed programs (defined as processes and modules) with added functionalities. Some calculi are available to combine superimpositions and to perform semantic checks so that the expected properties of the final program and the superimposed modules are verified. Our approach focuses on the around advice and is more usable by the end programmer since superimpositions require the programmer to define a great deal of semantics, which can make the approach difficult to understand and to use in practice.

Also, some core composition mechanisms are defined in the Composition Filter model [3]. These mechanisms rely on the definitions of workflows which define how filters interact. However, it is tricky to define a given workflow and 
make sure that it will be valid and usable in any case. Besides, the chosen approach makes the implementation quite difficult to read an maintain.

Finally, besides validation of aspect compositions, the strength of our approach comes from its simplicity: it can be straightforwardly mapped to a plain implementation, without having to use complex paradigms and API within the program.

\section{Conclusion}

In this paper, we study the Aspect Composition Issues (ACIs) when using the around advice construct, which is a significant construct for separating concerns, especially for AOP and related approaches. Our work defines a language called CompAr that allows the specification of composition-relevant information that includes boolean choices (forming the execution domain), action executions or invocations, and post-execution constraints. Our compiler then evaluates the specification within the defined domain and checks that all the execution paths fulfill the constraints.

Our study of the four real-life aspects (logging, authentication, persistence, and association) shows that our approach helps to detect and solve ACIs (see the persistence/association ACI of section 5). Besides, the fact that we define a new language makes the approach independent from existing concrete environments or languages. CompAr can then be used as a complementary tool or a DSL for helping the designers.

Finally, even though CompAr is a research prototype, our study is a proof of concept that validation of AO programs is possible. For instance, it would be possible, for a tool or language editor, to generate the abstract CompAr specification out of a real program. One could argue that we could face a state explosion problem when executing the specification (especially if we wanted to test all the possible orders). However, since the abstract specification focuses only on composition-relevant information, and that the number of around advice codes it somehow restrained in real systems, we think that this method is applicable in most cases.

We are currently working on several improvements of the language. For instance, we would like to introduce missing constructs such as exception catches, that have not been implemented yet. Modeling other kind of advice such as before, after, cflow, and call-located advice would be quite straightforward. Indeed, before and after advice are subcases of around once; cflow can be modeled by defining specific choices; call-located advice (in this paper we focus on executionlocated advice) can be modeled using a proxy-like design. More importantly, we also would like to enhance the post execution constraint sub-language to allow TLA-like expressions. This would allow the designer to specify advice where the performed actions must be executed in a certain order. For instance, a security aspect should always execute the crypt action before the decrypt action. 


\section{References}

1. AOP-Alliance. http://aopalliance.sf.net.

2. L. Bergmans, M. Aksit, and B. Tekinerdogan. Software Architectures and Component Technology, chapter Aspect Composition Using Composition Filters, pages 357-382. Kluwer Academic Publishers, 2001.

3. L. Bergmans and M. Aksits. Composing crosscutting concerns using composition filters. CACM, 44(10):51-57, 2001.

4. CompAr. http://www.lifl.fr/ pawlak/compar.

5. E. Dijkstra. A Discipline of Programming. Prentice Hall, 1976.

6. R. Douence, P. Fradet, and M. Südholt. Composition, reuse and interaction analysis of stateful aspects. In Proceedings of the 3rd Int. Conf. on Aspect-Oriented Software Development (AOSD'04), Mar. 2004.

7. M. Katara and S. Katz. Architectural views of aspects. In Proceedings of the 2nd international conference on Aspect-oriented software development, pages 1-10. ACM Press, 2003.

8. G. Kiczales, E. Hilsdale, J. Hugunin, M. Kersten, J. Palm, and W. Griswold. Getting started with AspectJ. Communications of the ACM, 44(10):59-65, 2001.

9. G. Kiczales, J. Lamping, A. Mendhekar, C. Maeda, C. Lopes, J. Loingtier, and J. Irwin. Aspect-oriented programming. In Proceedings of the European Conference on Object-Oriented Programming (ECOOP'97), 1997.

10. R. Lämmel. A semantical approach to method-call interception. In Proceedings of the 1st international conference on Aspect-oriented software development, pages 41-55. ACM Press, 2002.

11. M. Mezini and K. Ostermann. Conquering aspects with Caesar. In Proceedings of the 2nd International Conference on Aspect-Oriented Software Development (AOSD'03), 2003.

12. H. Ossher and P. Tarr. Using multidimensional separation of concerns to (re)shape evolving software. Communications of the ACM, 44(10):43-50, 2001.

13. D. L. Parnas. On the criteria to be used in decomposing systems into modules. Communications of the ACM, 15(12):1053-1058, 1972.

14. R. Pawlak, L. Duchien, and G. Florin. An automatic aspect weaver with a reflective programming language. In Proceedings of Reflection'99, July 1999.

15. R. Pawlak, L. Seinturier, L. Duchien, and G. Florin. Jac: A flexible solution for aspect-oriented programming in java. In Proceedings of Reflection 2001, LNCS 2192, pages 1-21, May 2001.

16. A. Popovici, G. Alonso, and T. Gross. Just-in-time aspects: Efficient dynamic weaving for java. In Proceedings of the 2nd international conference on AspectOriented Software Development, pages 100-109. ACM Press, 2003. 